\title{
Homozygous Inactivating Mutation in NANOS3 in Two Sisters with Primary Ovarian Insufficiency
}

\author{
Mariza G. Santos, ${ }^{1}$ Aline Z. Machado, ${ }^{1}$ Conceição N. Martins, ${ }^{1}$ Sorahia Domenice, \\ Elaine M. F. Costa, ${ }^{1}$ Mirian Y. Nishi, ${ }^{1}$ Bruno Ferraz-de-Souza, ${ }^{2}$ Soraia A. C. Jorge, ${ }^{3}$ \\ Carlos A. Pereira, ${ }^{3}$ Fernanda C. Soardi, ${ }^{4}$ Maricilda P. de Mello, ${ }^{4}$ Andrea T. Maciel-Guerra, ${ }^{5}$ \\ Gil Guerra-Junior, ${ }^{6}$ and Berenice B. Mendonca ${ }^{1}$ \\ ${ }^{1}$ Unidade de Endocrinologia do Desenvolvimento, Laboratório de Hormônios e Genética Molecular/LIM-42, \\ Hospital das Clínicas da Faculdade de Medicina da Universidade de São Paulo, Avenida Dr. Eneas de C Aguiar 155, 2 andar Bloco 6, \\ 05403-900 São Paulo, SP, Brazil \\ ${ }^{2}$ Laboratório de Carboidratos e Radioimunoensaios/LIM-18, Hospital das Clínicas da Faculdade de Medicina da Universidade de \\ São Paulo, Avenida Dr. Arnaldo 455, 01246-903 São Paulo, SP, Brazil \\ ${ }^{3}$ Laboratorio de Imunologia Viral, Instituto Butantan, Avenida Vital Brasil 1500, 05503-900 São Paulo, SP, Brazil \\ ${ }^{4}$ Centro de Biologia Molecular e Engenharia Genética/CBMEG, Faculdade de Ciências Médicas, Universidade Estadual de Campinas, \\ 13083-970 Campinas, SP, Brazil \\ ${ }^{5}$ Departamento de Genética Médica, Faculdade de Ciências Médicas, Universidade Estadual de Campinas, Rua Tessalia Vieira de \\ Camargo 126, 13083-970 Campinas, SP, Brazil \\ ${ }^{6}$ Departamento de Pediatria, Faculdade de Ciências Médicas, Universidade Estadual de Campinas, Rua Tessalia Vieira de \\ Camargo 126, 13083-970 Campinas, SP, Brazil
}

Correspondence should be addressed to Berenice B. Mendonca; beremen@usp.br

Received 10 March 2014; Revised 30 May 2014; Accepted 3 June 2014; Published 26 June 2014

Academic Editor: Svetlana Lajic

Copyright ( 2014 Mariza G. Santos et al. This is an open access article distributed under the Creative Commons Attribution License, which permits unrestricted use, distribution, and reproduction in any medium, provided the original work is properly cited.

Despite the increasing understanding of female reproduction, the molecular diagnosis of primary ovarian insufficiency (POI) is seldom obtained. The RNA-binding protein NANOS3 poses as an interesting candidate gene for POI since members of the Nanos family have an evolutionarily conserved function in germ cell development and maintenance by repressing apoptosis. We performed mutational analysis of NANOS3 in a cohort of 85 Brazilian women with familial or isolated POI, presenting with primary or secondary amenorrhea, and in ethnically-matched control women. A homozygous p.Glu120Lys mutation in NANOS 3 was identified in two sisters with primary amenorrhea. The substituted amino acid is located within the second C2HC motif in the conserved zinc finger domain of NANOS3 and in silico molecular modelling suggests destabilization of protein-RNA interaction. In vitro analyses of apoptosis through flow cytometry and confocal microscopy show that NANOS3 capacity to prevent apoptosis was impaired by this mutation. The identification of an inactivating missense mutation in NANOS3 suggests a mechanism for POI involving increased primordial germ cells (PGCs) apoptosis during embryonic cell migration and highlights the importance of NANOS proteins in human ovarian biology.

\section{Introduction}

Primary ovarian insufficiency (POI) is characterized by ovarian failure in women under the age of 40 years $[1,2]$. POI may present as primary amenorrhea $(\mathrm{PA})$ in severe cases with prepubertal onset or postpubertally as secondary amenorrhea (SA) associated with infertility, hypoestrogenism, and elevated gonadotropins (FSH $>40 \mathrm{U} / \mathrm{L}$ ). This complex spectrum of progressive ovarian abnormality is largely related to the size of primordial germ cells (PGCs) pool, where 
prepubertal onset might reflect a complete lack of germ cells since birth causing a failure in the maintenance of ovarian somatic structure and postpubertal onset would reflect a variably insufficient pool of oocytes. This disorder is associated with female infertility and affects 1 to $2 \%$ of all women [3-5].

Several genetic mechanisms may lead to POI, including chromosomal abnormalities of the X chromosome or autosomes and autoimmune disorders [6]. Despite the increasing understanding of female reproduction, defined causes or mechanisms resulting in primary ovarian insufficiency remain largely unknown [7]. Persani et al. have estimated the prevalence of known genetic alterations in POI patients originally classified as idiopathic to be around 20 to $25 \%$ of cases [8], whereas others have found this prevalence to be lower, around $10 \%$. Rare mutations have been described in genes involved in ovarian development and/or function such as FSHR (MIM 136435), LHCGR (MIM 152790), BMP15 (MIM 300247), POF1B (MIM 300603), NOBOX (MIM 610934), INHA (MIM 147380), GDF9 (MIM 601918), NR5A1 (MIM 184757), and FIGLA (MIM 608697) and in meiotic genes [9-23]. Nevertheless, mutations in these genes account for a minority of cases of ovarian dysfunction, indicating that additional factors remain to be identified.

Nanos was first identified in Drosophila, where it represses the translation of target mRNAs through binding to their $3^{\prime}$ UTR and has a conserved function in germ cell development across species. Members of the evolutionarily conserved Nanos gene family are preferentially expressed in the ovaries and are known to play an important role in germ cell development, maintenance, and survival [24-30]. In Drosophila, the single Nanos gene (Nos) is required for development of the abdomen as well as for germ line maintenance [31,32]. Three Nanos homologues exist in mouse, with Nanos 2 and Nanos 3 functioning primarily in male germ cell development and maintaining PGCs viability, respectively $[33,34]$. In mice, Nanos3 is expressed in the primordial germ cells (PGCs) from their formation until shortly after their appearance in the gonads (E13.5 in female and E14.5 in male embryos) [24]. Male and female mice deficient in Nanos 3 are infertile, and female $\mathrm{Nanos}^{-1-}$ mice have atrophic ovaries in which no germ cells are detectable due to loss of migrating PGCs during embryogenesis [24]. PGCs are lost by apoptosis in the absence of Nanos3, establishing an essential function of Nanos3 as a repressor of apoptosis in the germ cell [34].

A similar conserved function for NANOS proteins has been shown in humans. NANOS1 and NANOS2 seem to be mainly involved with male germ cell development and maintenance [35], and indeed NANOS1 mutations have been associated with male infertility manifested as nonobstructive azoospermia or oligozoospermia [36]. NANOS3, on the other hand, is expressed in both male and female fetal and adult gonads, and in vitro evidence suggests a pivotal function in human germ cell development [37].

Despite this compelling evidence for the importance of NANOS3 in germ cell maintenance, initial efforts to identify NANOS3 mutations in women with POI were not successful: in 2007, Qin et al. analyzed 168 infertile Caucasian and Chinese women and failed to identify pathogenic mutations
[38]. More recently, however, Wu et al. performed mutational analysis of the coding regions of NANOS1, NANOS2, and NANOS3 in 100 Chinese POI patients, identifying a heterozygous p.Arg153Trp NANOS3 mutation in a 23-year-old woman [39]. The mutant NANOS3 protein was shown to have decreased stability in vitro, leading the authors to postulate that reduced expression of NANOS3 in the ovaries would result in decreased PGC population and POI [39]. Herein we report a homozygous missense mutation in Nanos 3 found in two sisters from our cohort of 85 Brazilian women with POI and present supporting in vitro functional data.

\section{Materials and Methods}

2.1. Subjects. This study was approved by the ethics committee of Hospital das Clinicas, University of Sao Paulo School of Medicine, Brazil (Protocol number 1226/07).

Eighty-five patients with POI were referred to the Developmental Endocrinology Unit of Hospital das Clinicas, Sao Paulo, or to the Pediatric Department of University of Campinas, in Sao Paulo, Brazil, for evaluation of primary amenorrhea (45 patients, 10 familial cases) or secondary amenorrhea (40 unrelated patients). The age at diagnosis of amenorrhea was $29.2 \pm 5.9$ years (18 to $39 \mathrm{yrs}$ ). Parental consanguinity was present in $80 \%$ of familial cases, and no other relevant familial history was reported. Each family had 2 affected individuals, and age at diagnosis of familial cases was $19.4 \pm 6.2$ years (14 to 36 yrs). FSH levels were elevated in all patients, ranging from 27 to $150 \mathrm{U} / \mathrm{L}$ in patients with primary amenorrhea and from 32 to $158 \mathrm{U} / \mathrm{L}$ in patients with secondary amenorrhea. Mutations in FSHR, NR5A1, BMP15, and GDF9, premutations in FMR1, and thyroid, adrenal, or ovarian autoimmune disorders had been excluded in all patients. Ethnically and age-matched (23 to 39 years old) women with normal menarche and menstrual cycles were invited to participate as controls. Institutional review board approval and written informed consent were obtained from all subjects before sample collection for DNA analysis.

2.2. Mutational Analysis of NANOS3. Two protein-coding transcripts of Nanos3 are described in the Ensembl database: a 581 bp transcript (ENST00000339133) resulting in a 192residue protein and a $776 \mathrm{bp}$ transcript (ENST00000397555) resulting in a 173-residue protein; only transcript ENST00000339133 is part of the human consensus coding sequence set (CCDS).

Following genomic DNA extraction from peripheral blood leukocytes, the entire exonic region and at least $15 \mathrm{bp}$ of exon/intron boundaries of transcript ENST00000339133 (RefSeq NM_001098622.2) were PCR-amplified and submitted to direct automated sequencing in an ABI PRISM 310 (Applied Biosystems, Foster City, CA).

2.3. Apoptosis Detection by Flow Cytometry. A pCMV6AC-GFP-NANOS3 expression vector (OriGene Technologies, Rockville, $\mathrm{MD}$ ) was used as a template to generate a c.358G > A mutant using the QuikChange site-directed mutagenesis kit (Stratagene, La Jolla, CA) and specific primers 


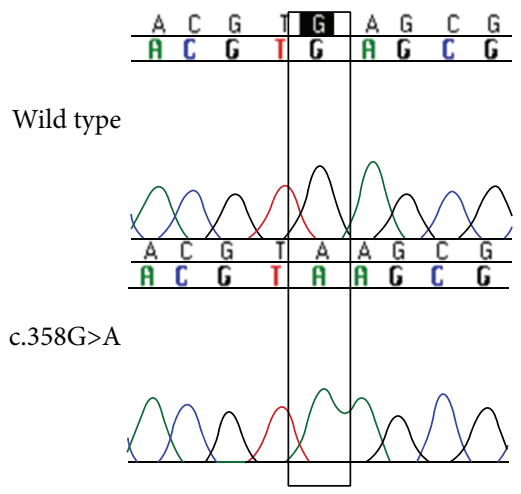

(a)

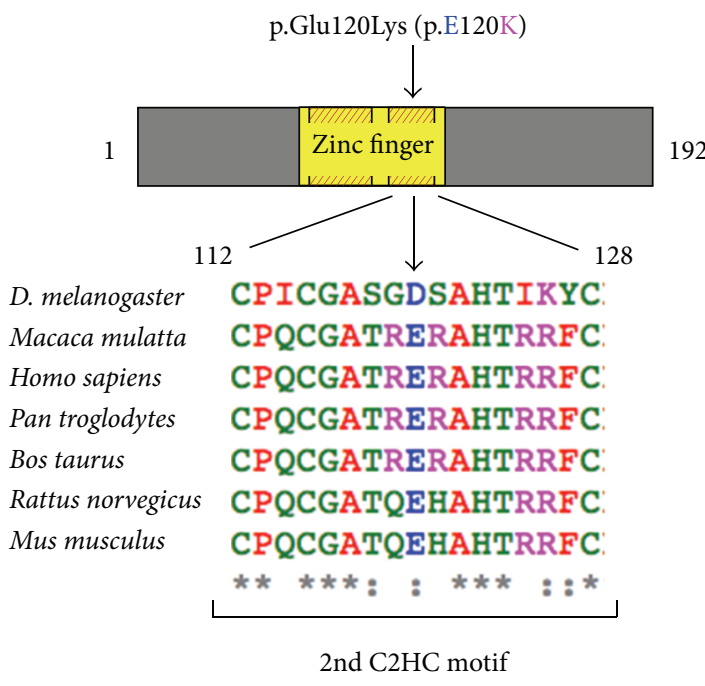

(b)

FIGURE 1: A Mutational analysis of NANOS3 revealed a homozygous c.358G>A substitution in exon 1 in two sisters with primary amenorrhea. The GAG to AAG change in codon 120 results in a substitution from glutamic acid to lysine in the codified protein (p.Glu120Lys). B, Cartoon representation of the NANOS3 protein (192 amino acids), with the zinc finger domain (amino acid 76 to 130) depicted in yellow, showing the location of the p.Glu120Lys mutation. Glutamic acid 120 (shown as E120) is localized within one of two highly conserved Cys-Cys-His-Cys $(\mathrm{C} 2 \mathrm{HC})$ motifs (shaded red and yellow boxes). Conservation among species of the second C2HC motif sequence (human residues 112 to 128 ) is shown in detail; glutamic acid 120 (E) is highly conserved. Alignment was performed in Clustal Omega using protein sequences obtained from UniProt.

(F: 5' GGC GCC ACA CGT AAG CGC GCC CAC AC 3'; R: $5^{\prime}$ GTG TGG GCG CGC TTA CGT GTG GCG CC $\left.3^{\prime}\right)$. African green monkey kidney COS-1 cells were transiently transfected using Lipofectamine 2000 (Invitrogen, Carlsbad, CA) and analyzed 48 hours later with or without treatment with $5 \mathrm{mM}$ sodium butyrate (Calbiochem, San Diego, CA). Cells were stained with Annexin- $V$ conjugates (Molecular Probes Invitrogen, Carlsbad, CA) according to manufacturer's protocol. GFP and Annexin-V fluorescence were excited at $488 \mathrm{~nm}$ and $650 \mathrm{~nm}$ and emission measured using $530 / 30 \mathrm{~nm}$ and $660 / 30$ band pass filters, respectively.

2.4. Confocal Microscopy. Confocal microscopy micrographs were obtained using a $488 \mathrm{~nm}$ laser line and light emitted between 500 and $600 \mathrm{~nm}$ for GFP detection and a $650 \mathrm{~nm}$ laser line and light emitted between 660 and $700 \mathrm{~nm}$ for red Annexin-V detection. Images were collected on a META LSM 510 laser scanning confocal microscope equipped with a $63.0 \times 1.2 \mathrm{~W}$ objective (Carl Zeiss, Jena, Germany). The same settings for image acquisition and processing were applied for all samples to allow comparison of the fluorescence intensities among different samples.

2.5. Molecular Modeling of NANOS3. A model for mutated human NANOS3 was built using the resolved structure of L3MBTL1 (PDB-ID 2RI7) as template since NANOS3 structure has not yet been resolved. In order to find the best model for NANOS3, a Blast alignment with proteins available in PDB was performed. Highest homology was found with the crystal structure of Nanos from zebrafish (PDB-ID 3ALR) with 58\% identity. However, this structure did not include the region where the p.Glu120Lys mutation is located. Therefore, we looked for another protein bearing similarities with NANOS3. Such protein was L3MBTL1. Its X-ray resolved structure presented the second highest homology with NANOS3 (33\% of identity) and included the p.Glu120Lys mutant region. Molecular modeling was performed with the SwissModel web-served program and images examined and edited using the web-based BlueStar STING (CNPTIA-Embrapa, Brazil) and PyMOL softwares (freely available at http://www.pymol.org).

\section{Results}

3.1. Identification of NANOS3 Mutation. Mutational analysis of NANOS3 revealed that two sisters carried a homozygous c.358G $>$ A, p.Glu120Lys mutation (Figure 1), which was not detected in 113 ethnically matched control women and has not been reported in the 1000 Genome Project or dbSNP databases. Their mother was heterozygous and the father's DNA was not available for study. In the remaining 83 women composing our POI cohort, no new variants were identified in NANOS3. Two previously described SNPs were identified in our cohort, rs897790 and rs2016163 (Table 1).

The patients carrying the homozygous c.358G $>$ A mutation were from the northeast region of Brazil (Piauí State) and born to nonconsanguineous parents. At 12 and 16 years old they were evaluated for breast underdevelopment. Both girls had not reached menarche, and physical examination revealed Tanner stage I breast development and Tanner stage III pubic hair. Basal gonadotropin levels were elevated with predominantly high FSH levels $(\mathrm{LH}=63$ and $21 \mathrm{U} / \mathrm{L}$, 
TABLE 1: Polymorphic variants in NANOS3 identified in POI patients and controls.

\begin{tabular}{|c|c|c|c|c|c|c|c|}
\hline \multirow{3}{*}{ Variant } & \multirow{3}{*}{ dbSNP id } & \multicolumn{6}{|c|}{ Genotype frequency (\%) } \\
\hline & & \multicolumn{3}{|c|}{ Patients with POI } & \multicolumn{3}{|c|}{ Controls } \\
\hline & & Wild-type & Heterozygote & Homozygote & Wild type & Heterozygote & Homozygote \\
\hline c. $-23 \mathrm{C}>\mathrm{T}\left(5^{\prime} \mathrm{UTR}\right)$ & rs897790 & 60 & 34.1 & 5.9 & 72.5 & 25.7 & 1.8 \\
\hline c. $353 \mathrm{~A}>\mathrm{G}$ (exon 1 , synonymous) & rs2016163 & 50.6 & 40 & 9.4 & 70 & 27.4 & 2.6 \\
\hline
\end{tabular}

FSH $=150$ and $61 \mathrm{U} / \mathrm{L}$, resp.). Estradiol levels were within the prepubertal range in both girls. Measured heights were 145 and $156 \mathrm{~cm}$, and weights were 38 and $46 \mathrm{Kg}$, respectively. Pelvic ultrasonography showed no masses, and ovaries were not visualized. Treatment with conjugated estrogens followed by progesterone replacement resulted in complete breast development and normal cycling.

Their heterozygous mother has a history of menarche at 16 years old and difficulties to conceive. Her first pregnancy was at 27 years old after 4 years of attempts to conceive and her second and last gestation occurred at 30. Menopause occurred at 51 years of age.

3.2. Increased Apoptosis In Vitro with p.Glu120Lys Mutant NANOS3. Considering that Nanos3 has been shown to maintain germ cell development in model organisms through repression of apoptosis [34], in vitro studies were performed to assess the effects of the p.Glu120Lys substitution on the apoptotic rate of cultured cells. COS-1 cells were transfected with GFP-tagged wild-type or mutant NANOS3 and viability assessed by Annexin-V staining. Flow cytometry analysis showed that the percentage of Annexin-V stained GFPpositive cells was significantly higher in cells transfected with mutant NANOS3 in comparison to wild type, in untreated or sodium butyrate-treated cells $(P<0.05)$ (Figure 2$)$, indicating that the p.Glu120Lys substitution profoundly impairs cell viability.

Since NANOS3 has been shown to act in the cell nucleus [37], studies were performed to investigate whether the p.Glu120Lys mutation would affect intracellular localization. Transfected COS-1 cells were analyzed by confocal laser scanning microscopy for cellular localization of GFP-tagged wild type or mutant NANOS3 and Annexin-V staining (Figure 3). Nuclear localization was observed in cells transfected with both wild type and mutant cDNA. However, Annexin-V staining in the plasma membrane, indicating apoptosis, was only detected in cells expressing the p.Glu120Lys mutant NANOS3.

3.3. In Silico Mutant NANOS3 Modelling. Glutamic acid120 is a negatively charged amino acid located within the zinc finger domain. This residue is found at the surface of NANOS3 within the second Cys-Cys-His-Cys (C2HC) motif, which is important for its ability to bind RNA [40]. In general, substitution of amino acids that share similar hydrogen binding capabilities and tridimensional requirements such as glutamic acid and lysine has limited structural consequences. However, lysine has a positive charge on the aliphatic side chain that can affect DNA binding activity when inserted

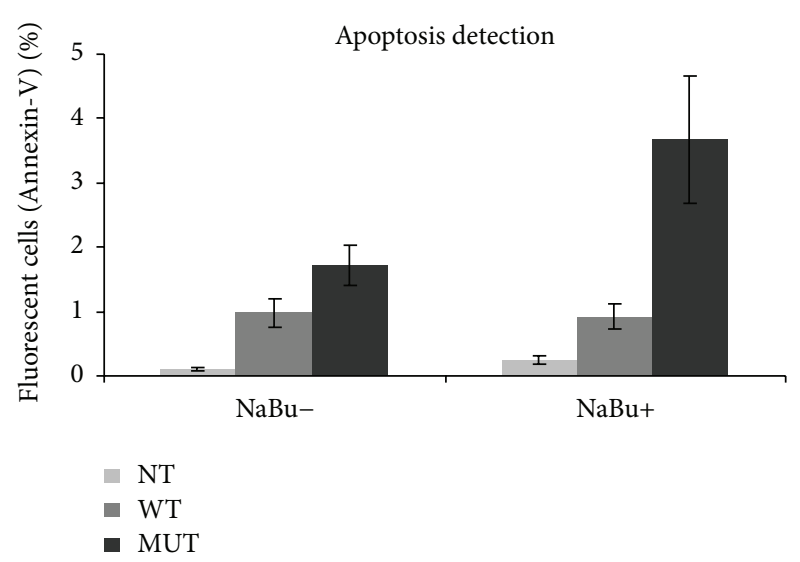

FIGURE 2: Detection of apoptosis using flow cytometry and Annexin-V staining in COS-1 cells transfected with wild type (WT) or p.Glu120Lys mutant (MUT) NANOS3-GFP, before and after induction of apoptosis with sodium butyrate $(\mathrm{NaBu})$. Expression of mutant NANOS3 results in significantly higher apoptosis in comparison to wild type $(P<0.05)$. Error bars represent SEM for nine replicates; NT: nontransfected control.

into the zinc finger domain. In silico analysis shows that lysine 120 establishes a new contact with Lys96 and abolishes the original contact with His123 (Figure 4). Consequently, these changes could lead to internal contact conformational modifications resulting in impaired protein function. In addition, 3D structure indicates that the negatively charged glutamic acid 120 residue is surrounded by negatively charged residues (Figure 5). Therefore, the presence of the positively charged lysine 120 residue in the protein surface might cause strong electrostatic repulsion among the side chains and lead to destabilization upon protein-RNA interaction.

\section{Discussion}

The present study aimed to investigate the contribution of variants in NANOS3 to human POI. We have identified a nonsynonymous homozygous NANOS3 mutation in two sisters with primary amenorrhea.

During development, once germ cells populate the gonads, they are exposed to several apoptotic waves. NANOS3 plays an important role in the maintenance and survival of primordial germ cells and therefore constitutes an interesting candidate gene for nonsyndromic POI. Indeed, NANOS3 has been shown to function in germ cell development in a breadth of organisms, from flies and worms through frogs and mammals. In mice, loss of Nanos 3 results 

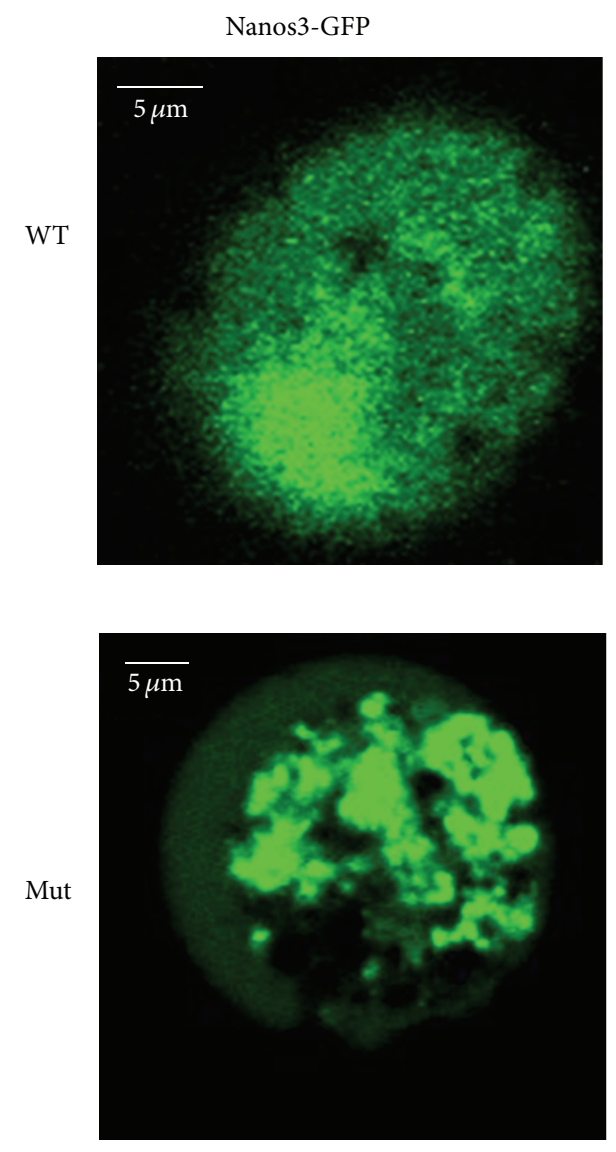

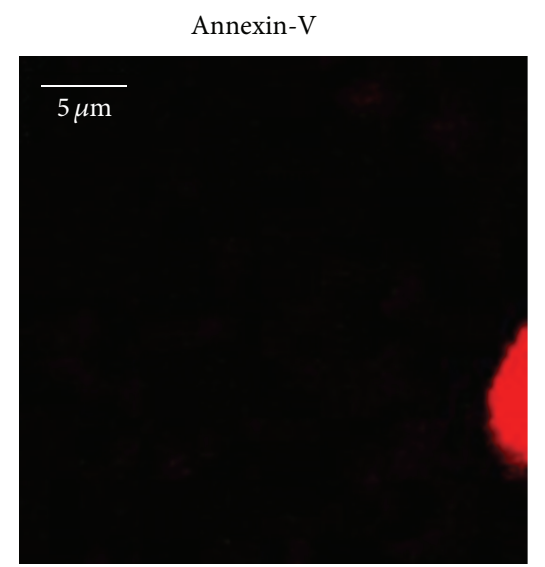

(a)

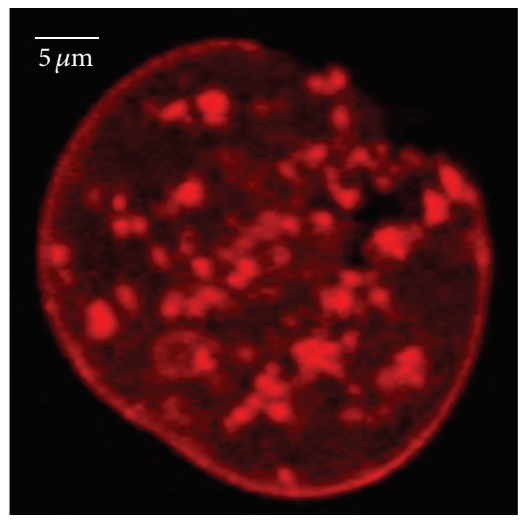

(b)
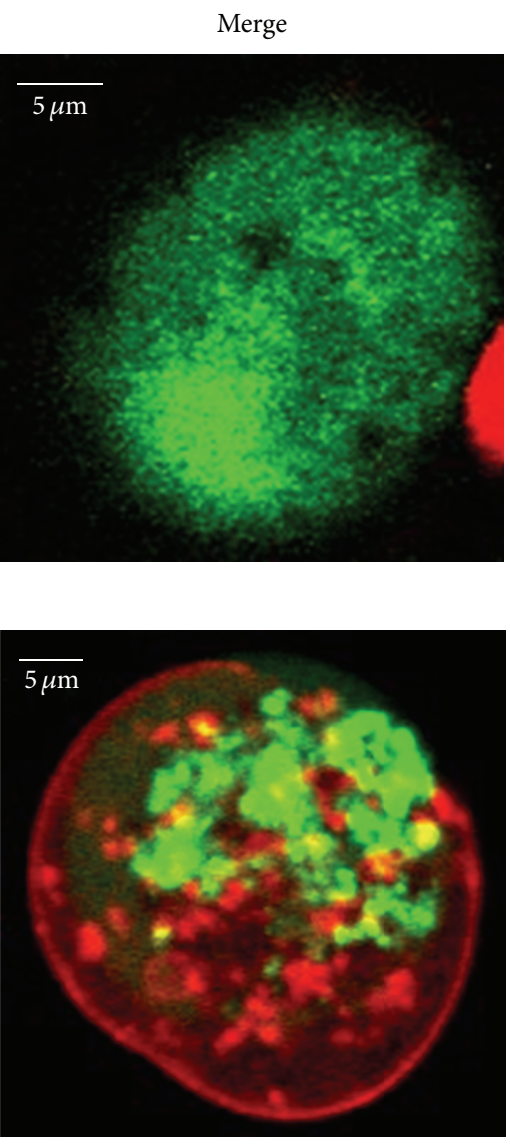

FIGURE 3: Confocal microscopy analysis of apoptosis in COS-1 cells following $48 \mathrm{~h}$ induction of apoptosis with sodium butyrate. In cells transfected with wild type (WT, upper row) or p.Glu120Lys mutant (Mut, lower row) GFP-tagged NANOS3, expression of green NANOS3GFP is seen predominantly in the nucleus. Red Annexin-V staining in the cytoplasm and plasma membrane, indicating apoptosis, is seen in cells expressing p.Glu120Lys mutant NANOS3 but not in those expressing WT NANOS3.
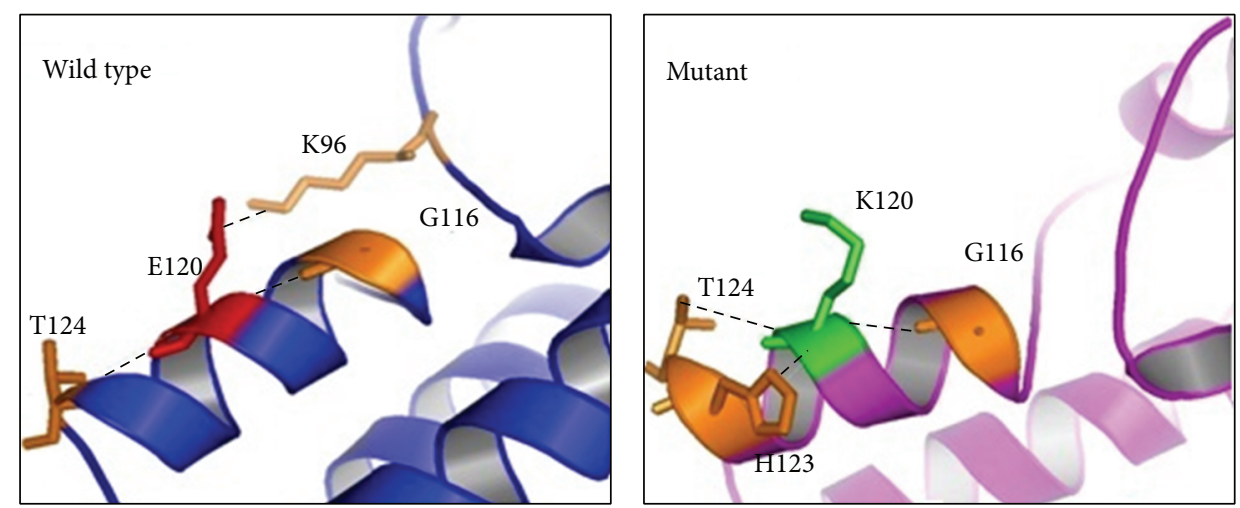

FIGURE 4: In silico analysis of amino acid interaction modification shows the different conformation conferred by the p.Glu120Lys substitution. Glutamic acid 120 (shown as E120) makes a hydrophobic interaction with lysine 96 (K96) and hydrogen bonds with both glycine 116 (G116) and threonine 124 (T124). The substitution to lysine 120 (K120) modifies structural points of contact establishing a new interaction with histidine 123 (His123) through aromatic stacking and abolishes the hydrophobic interaction with K96. 


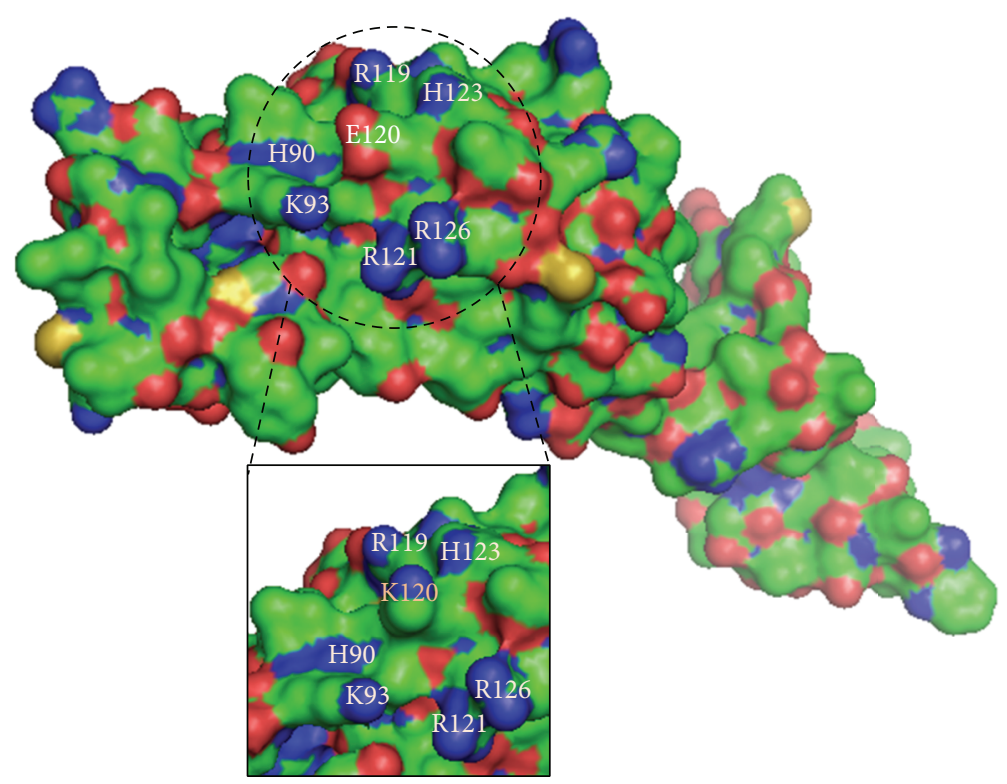

FIGURE 5: Bulk representation of wild-type NANOS3, with p.Glu120Lys mutant shown in details box. The dashed circle highlights a protein surface region rich in basic residues, shown in blue. At the center of this region, lies the acidic residue glutamic acid 120 (E120), shown in red. In the details, substitution by lysine 120 (K120) disturbs the electrostatic interactions among adjacent residues.

in infertility due to apoptosis of migrating PGCs during fetal life, resulting in atrophic gonads where germ cells are absent $[24,34]$. Furthermore, the contribution of Nanos3 to PGC maintenance and survival seems to be dosage-dependent in mice, as a model with attenuated Nanos3 transcript levels displayed markedly reduced numbers of PGCs [39].

In humans, NANOS3 is expressed in fetal and adult ovaries during multiple stages of oogenesis and in vitro studies of human embryonic stem cell-derived germ cells have shown coexpression of NANOS3 in nuclei with important germ cell factors such as BLIMP1, VASA, and STELLA in the cytoplasm [37]. In these cells, reduction of NANOS3 expression resulted in altered gene expression patterns and reduced number of cells in active division, suggesting that in humans too NANOS3 has an important function in germ cell maintenance and survival. Recently, a heterozygous p.Arg153Trp NANOS3 mutation was reported in a 23 -yearold Chinese woman out of a cohort of 100 women with POI [39]. Familial segregation was not defined, but the mutation was not found in 200 ethnically-matched controls. In vitro studies suggested decreased protein stability due to the p.Arg153>Trp mutation, leading the authors to postulate a mechanism of reduced dosage of NANOS3 expression in the ovaries leading to decreased PGC population and resulting in POI [39].

The p.Glu120Lys mutation identified in our patients lies within the NANOS3 zinc finger domain, which is composed of two C2HC motifs located between amino acids 77 and 131. The differences in chemical properties between amino acids might lead to impaired binding of NANOS 3 to its mRNA targets, as suggested by protein modeling. In vitro experiments show that the mutant NANOS3 identified in the two sisters with POI was associated with increased apoptosis in transfected cells, suggesting that loss of NANOS3-mediated protective effect against apoptosis in primordial germ cells may be the mechanism underlying POI in these patients.

Primordial germ cell pool size in the developing ovary and maintenance of these cells throughout life are at the center of the pathogenesis of POI. It is remarkable that our two patients with homozygous NANOS3 mutation have presented with primary amenorrhea, perhaps indicating more severely PGC-depleted ovaries, while their heterozygous mother had difficulty to conceive, which could represent a manifestation of insufficient PGC pool size. Indeed, the patient described by $\mathrm{Wu}$ et al. carried a heterozygous missense change and presented with secondary, rather than primary, amenorrhea [39], supporting the concept of a dosage effect for NANOS3related POI.

In conclusion, we report a rare homozygous missense mutation in NANOS3 in two Brazilian sisters with primary amenorrhea from a cohort of 85 Brazilian women with POI. In vitro and in silico functional studies support that this inactivating mutation abolishes NANOS3 ability to prevent apoptosis, suggesting a mechanism for POI involving increased PGC apoptosis during embryonic cell migration.

\section{Conflict of Interests}

The authors declare that there is no conflict of interests regarding the publication of this paper.

\section{Acknowledgments}

The authors thank patients and their families for participating in this study. They are grateful to Jorge Mario C F 
Junior (Flow Cytometry Laboratory, Instituto Butantan) and Toshie Kawano and Alexsander Seixas de Souza (Parasitology Laboratory, Instituto Butantan) for technical assistance. This work was supported by Conselho Nacional de Desenvolvimento Científico e Tecnológico (CNPq, Grant 301339/20089) and the São Paulo Research Foundation (FAPESP, Grants 05/04726-0, 07/512156, and 10/51102-0).

\section{References}

[1] C. K. Welt, "Primary ovarian insufficiency: a more accurate term for premature ovarian failure," Clinical Endocrinology, vol. 68, no. 4, pp. 499-509, 2008.

[2] L. M. Nelson, "Clinical practice. Primary ovarian insufficiency," The New England Journal of Medicine, vol. 360, no. 6, pp. 606$614,2009$.

[3] D. Goswami and G. S. Conway, "Premature ovarian failure," Human Reproduction Update, vol. 11, no. 4, pp. 391-410, 2005.

[4] L. M. Nelson, S. N. Covington, and R. W. Rebar, "An update: spontaneous premature ovarian failure is not an early menopause," Fertility and Sterility, vol. 83, no. 5, pp. 1327-1332, 2005.

[5] P. Beck-Peccoz and L. Persani, "Premature ovarian failure," Orphanet Journal of Rare Diseases, vol. 1, no. 1, article 9, 2006.

[6] D. Toniolo, "X-linked premature ovarian failure: a complex disease," Current Opinion in Genetics and Development, vol. 16, no. 3, pp. 293-300, 2006.

[7] M. M. Matzuk and D. J. Lamb, “The biology of infertility: research advances and clinical challenges," Nature Medicine, vol. 14, no. 11, pp. 1197-1213, 2008.

[8] L. Persani, R. Rossetti, and C. Cacciatore, "Genes involved in human premature ovarian failure," Journal of Molecular Endocrinology, vol. 45, no. 5, pp. 257-279, 2010.

[9] P. Laissue, G. Vinci, R. A. Veitia, and M. Fellous, "Recent advances in the study of genes involved in non-syndromic premature ovarian failure," Molecular and Cellular Endocrinology, vol. 282, no. 1-2, pp. 101-111, 2008.

[10] R. Rossetti, E. D. Pasquale, A. Marozzi et al., "BMP15 mutations associated with primary ovarian insufficiency cause a defective production of bioactive protein," Human Mutation, vol. 30, no. 5, pp. 804-810, 2009.

[11] Y. Qin, Y. Choi, H. Zhao, J. L. Simpson, Z. Chen, and A. Rajkovic, "NOBOX homeobox mutation causes premature ovarian failure," The American Journal of Human Genetics, vol. 81, no. 3, pp. 576-581, 2007.

[12] D. Toniolo and F. Rizzolio, "X chromosome and ovarian failure," Seminars in Reproductive Medicine, vol. 25, no. 4, pp. 264-271, 2007.

[13] A. Lacombe, H. Lee, L. Zahed et al., "Disruption of POF1B binding to nonmuscle actin filaments is associated with premature ovarian failure," American Journal of Human Genetics, vol. 79, no. 1, pp. 113-119, 2006.

[14] A. N. Shelling, K. A. Burton, A. L. Chand et al., "Inhibin: a candidate gene for premature ovarian failure," Human Reproduction, vol. 15, no. 12, pp. 2644-2649, 2000.

[15] A. Marozzi, C. Porta, W. Vegetti et al., "Mutation analysis of the inhibin alpha gene in a cohort of Italian women affected by ovarian failure," Human Reproduction, vol. 17, no. 7, pp. 1741$1745,2002$.
[16] H. Dixit, L. K. Rao, V. V. Padmalatha et al., "Missense mutations in the BMP15 gene are associated with ovarian failure," Human Genetics, vol. 119, no. 4, pp. 408-415, 2006.

[17] P. Laissue, S. Christin-Maitre, P. Touraine et al., "Mutations and sequence variants in GDF9 and BMP15 in patients with premature ovarian failure," European Journal of Endocrinology, vol. 154, no. 5, pp. 739-744, 2006.

[18] E. Kovanci, J. L. Simpson, P. Amato et al., "Oocyte-specific Gprotein-coupled receptor 3 (GPR3): no perturbations found in 82 women with premature ovarian failure (first report)," Fertility and Sterility, vol. 90, no. 4, pp. 1269-1271, 2008.

[19] X. X. Zhao, N. Suzumori, M. Yamaguchi, and K. Suzumori, "Mutational analysis of the homeobox region of the human NOBOX gene in Japanese women who exhibit premature ovarian failure," Fertility and Sterility, vol. 83, no. 6, pp. 18431844, 2005.

[20] D. Loureņo, R. Brauner, L. Lin et al., "Mutations in NR5A1 associated with ovarian insufficiency," The New England Journal of Medicine, vol. 360, no. 12, pp. 1200-1210, 2009.

[21] B. Mandon-Pépin, P. Touraine, F. Kuttenn et al., "Genetic investigation of four meiotic genes in women with premature ovarian failure," European Journal of Endocrinology, vol. 158, no. 1, pp. 107-115, 2008.

[22] H. Zhao, Z. Chen, Y. Qin et al., "Transcription factor FIGLA is mutated in patients with premature ovarian failure," American Journal of Human Genetics, vol. 82, no. 6, pp. 1342-1348, 2008.

[23] Z. Z. Zhao, J. N. Painter, J. S. Palmer et al., "Variation in bone morphogenetic protein 15 is not associated with spontaneous human dizygotic twinning," Human Reproduction, vol. 23, no. 10, pp. 2372-2379, 2008.

[24] M. Tsuda, Y. Sasaoka, M. Kiso et al., "Conserved role of nanos proteins in germ cell development," Science, vol. 301, no. 5637, pp. 1239-1241, 2003.

[25] L. Mosquera, C. Forristall, Y. Zhou, and M. L. King, "A mRNA localized to the vegetal cortex of Xenopus oocytes encodes a protein with a nanos-like zinc finger domain," Development, vol. 117, no. 1, pp. 377-386, 1993.

[26] M. Pilon and D. A. Weisblat, "A nanos homolog in leech," Development, vol. 124, no. 9, pp. 1771-1780, 1997.

[27] K. Subramaniam and G. Seydoux, "nos-1 and nos-2, two genes related to Drosophila nanos, regulate primordial germ cell development and survival in Caenorhabditis elegans," Development, vol. 126, no. 21, pp. 4861-4871, 1999.

[28] A. Forbes and R. Lehmann, "Nanos and Pumilio have critical roles in the development and function of Drosophila germline stem cells," Development, vol. 125, no. 4, pp. 679-690, 1998.

[29] M. Köprunner, C. Thisse, B. Thisse, and E. Raz, "A zebrafish nanos-related gene is essential for the development of primordial germ cells," Genes \& Development, vol. 15, no. 21, pp. 2877$2885,2001$.

[30] H. Kurokawa, Y. Aoki, S. Nakamura, Y. Ebe, D. Kobayashi, and M. Tanaka, "Time-lapse analysis reveals different modes of primordial germ cell migration in the medaka Oryzias latipes," Development Growth and Differentiation, vol. 48, no. 3, pp. 209221, 2006.

[31] S. Kobayashi, M. Yamada, M. Asaoka, and T. Kitamura, "Essential role of the posterior morphogen nanos for germline development in Drosophila," Nature, vol. 380, no. 6576, pp. 708711, 1996

[32] H. Sano, M. Mukai, and S. Kobayashi, "Maternal Nanos and Pumilio regulate zygotic vasa expression autonomously in the 
germ-line progenitors of Drosophila melanogaster embryos," Development Growth \& Differentiation, vol. 43, no. 5, pp. 545$552,2001$.

[33] A. Suzuki and Y. Saga, "Nanos2 suppresses meiosis and promotes male germ cell differentiation," Genes \& Development, vol. 22, no. 4, pp. 430-435, 2008.

[34] H. Suzuki, M. Tsuda, M. Kiso, and Y. Saga, "Nanos3 maintains the germ cell lineage in the mouse by suppressing both Baxdependent and -independent apoptotic pathways," Developmental Biology, vol. 318, no. 1, pp. 133-142, 2008.

[35] K. M. Kusz, L. Tomczyk, M. Sajek et al., "The highly conserved NANOS2 protein: Testis-specific expression and significance for the human male reproduction," Molecular Human Reproduction, vol. 15, no. 3, pp. 165-171, 2009.

[36] K. Kusz-Zamelczyk, M. Sajek, A. Spik et al., "Mutations of NANOS1, a human homologue of the Drosophila morphogen, are associated with a lack of germ cells in testes or severe oligoastheno-teratozoospermia," Journal of Medical Genetics, vol. 50, no. 3, pp. 187-193, 2013.

[37] V. T. Julaton and R. A. Reijo Pera, "NANOS function in human germ cell development," Human Molecular Genetics, vol. 20, no. 11, pp. 2238-2250, 2011.

[38] Y. Qin, H. Zhao, E. Kovanci, J. L. Simpson, Z. Chen, and A. Rajkovic, "Mutation analysis of NANOS3 in 80 Chinese and 88 Caucasian women with premature ovarian failure," Fertility \& Sterility, vol. 88, no. 5, pp. 1465-1467, 2007.

[39] X. Wu, B. Wang, Z. Dong et al., "A NANOS3 mutation linked to protein degradation causes premature ovarian insufficiency," Cell Death and Disease, vol. 4, article e825, 2013.

[40] H. Hashimoto, K. Hara, A. Hishiki et al., "Crystal structure of zinc-finger domain of Nanos and its functional implications," EMBO Reports, vol. 11, no. 11, pp. 848-853, 2010. 


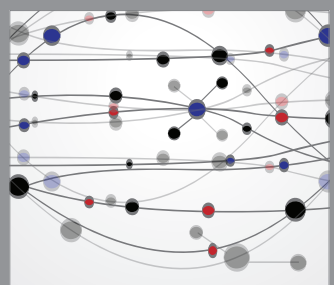

The Scientific World Journal
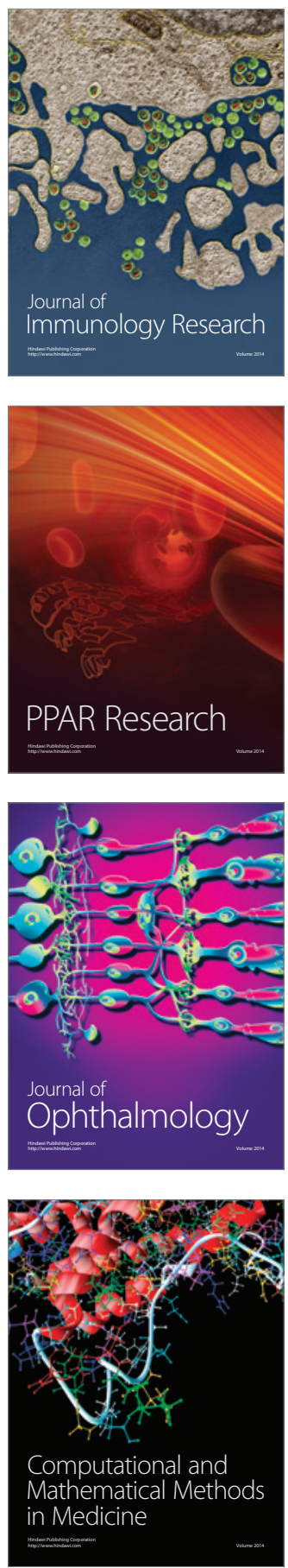

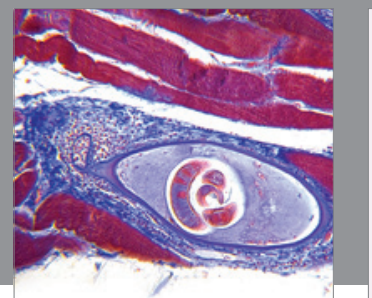

Gastroenterology

Research and Practice
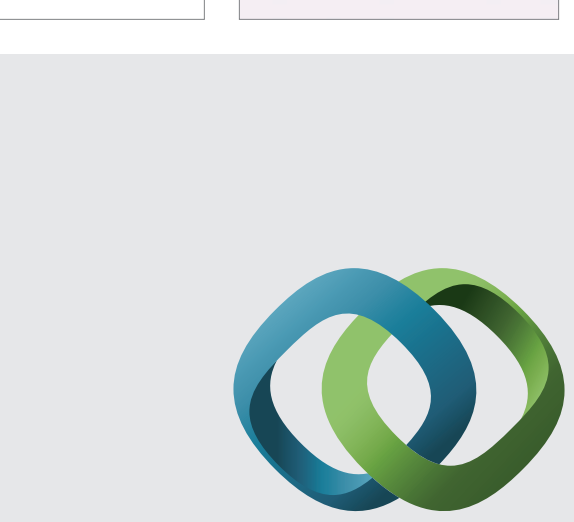

\section{Hindawi}

Submit your manuscripts at

http://www.hindawi.com
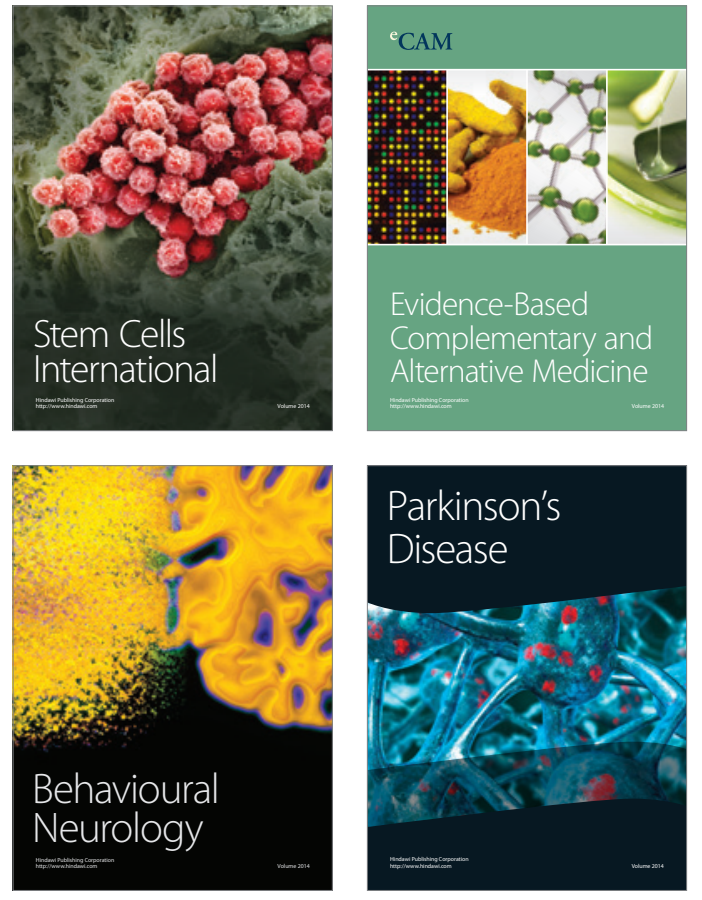
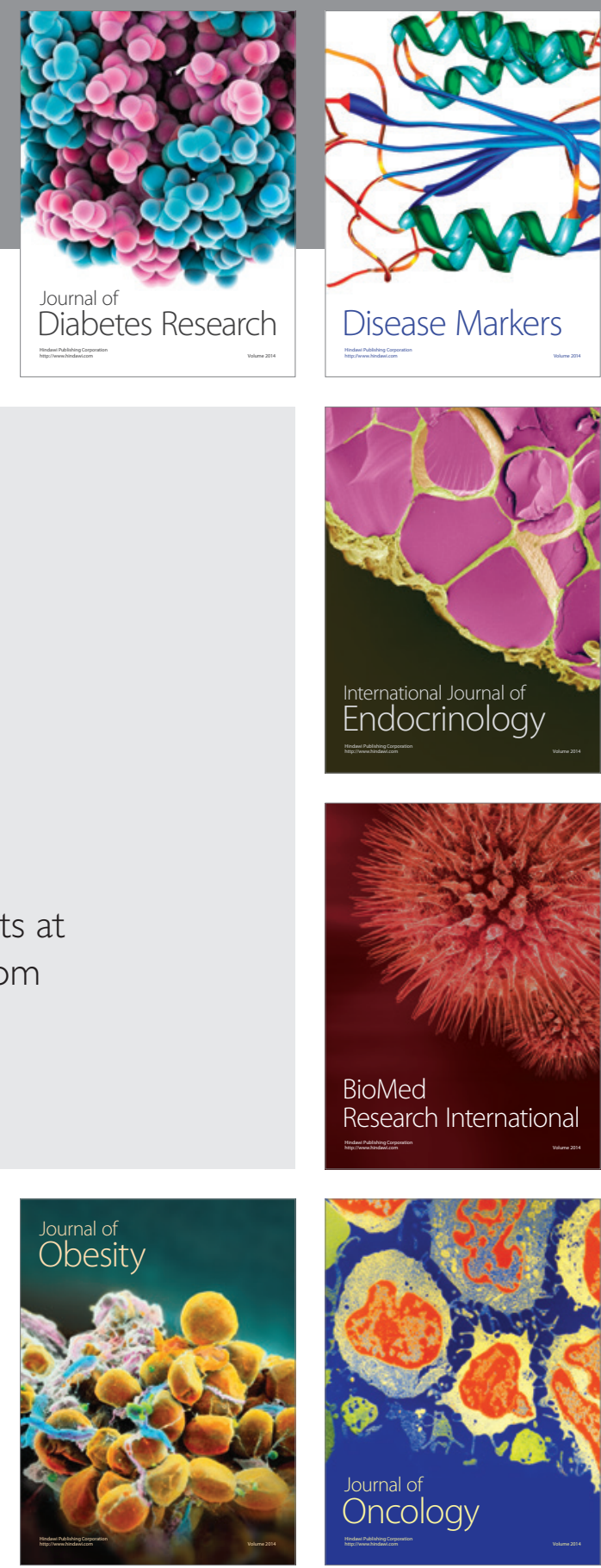

Disease Markers
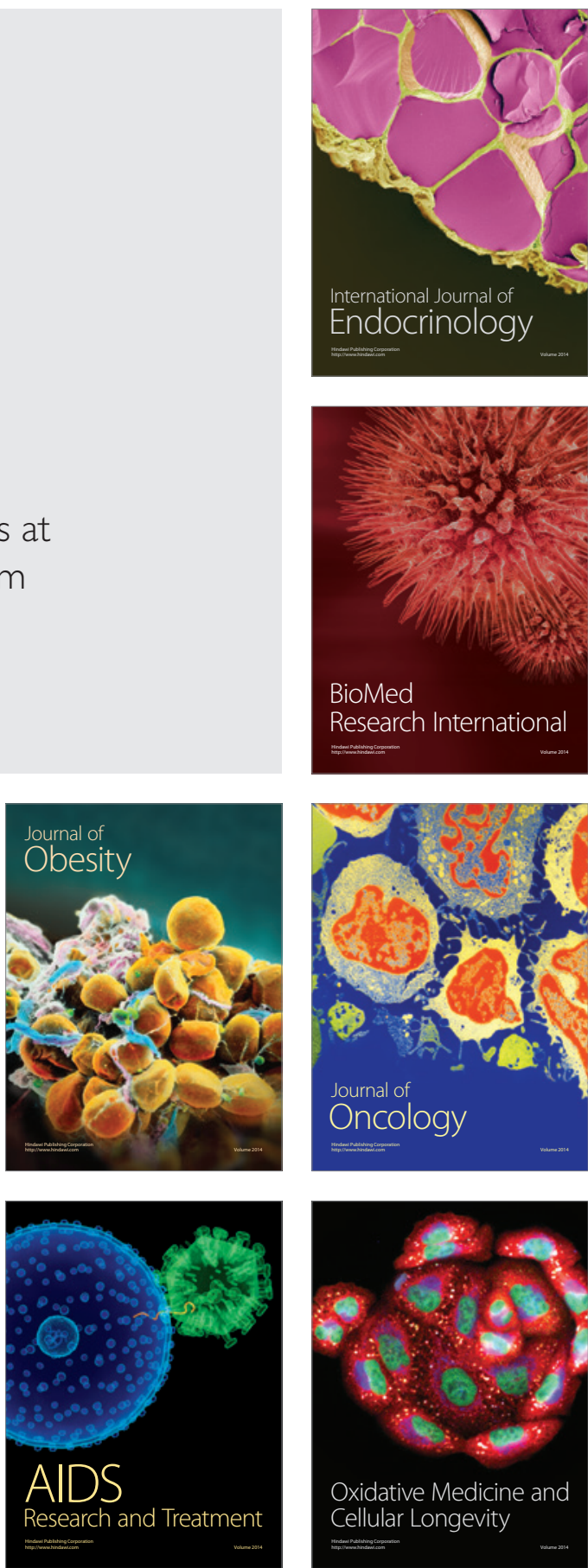